\title{
Potent and selective inhibitors of class A $\beta$-lactamase: 7-prenyloxy coumarins
}

\author{
Hadi Safdari ${ }^{1}$, Alireza Neshani ${ }^{2}$, Ali Sadeghian ${ }^{3}$, Masoomeh Ebrahimi ${ }^{2}$, Mehrdad Iranshahi $^{4}$ and \\ Hamid Sadeghian ${ }^{1,3}$
}

Class $A$ and $D$-lactamases are the main causes of resistance against $\beta$-lactam antibiotics, especially the penam group, in Staphylococcus aureus. On the basis of the potentiator property of ethanolic extracts of Ferula szowitsiana root on penicillin, MIC values observed for resistant $S$. aureus, the main naturally occurring compounds in these extracts, auraptene, umbelliprenin and galbanic acid, were evaluated for $\beta$-lactamase inhibitory activity. Amongst them auraptene showed the most potent inhibitory activity (IC50 $=21 \pm 1.5 \mu \mathrm{M})$ toward class $A \beta$-lactamase, whereas no inhibition was observed for class $D$ $\beta$-lactamase. To obtain the structure activity relationship of the mentioned compounds and rationalize the enzyme inhibitory results, docking analysis was performed for both groups of $\beta$-lactamases. Docking analysis showed that the compounds have 100-500-fold lower bonding affinity toward the class D $\beta$-lactamase than toward the class A enzyme.

The Journal of Antibiotics (2014) 67, 373-377; doi:10.1038/ja.2014.9; published online 12 February 2014

Keywords: auraptene; docking; Ferula szowitsiana; penicillin; Staphylococcus aureus

\section{INTRODUCTION}

Staphylococcus aureus is now the major cause of nosocomial pneumonia and surgical infections, and is the most common source of nosocomial bloodstream infections behind coagulase-negative staphylococci. ${ }^{1}$ Infections and outbreaks in nursing homes ${ }^{2,3}$ and among outpatient populations $s^{4,5}$ are common throughout the world. Since the introduction of antibiotics against bacterial infections (from 1940), microorganisms have shown a substantial ability to protect themselves by creating and acquiring antibiotic resistance. By 1942, penicillin resistance was observed in $S$. aureus after 3 months of clinical trials. ${ }^{6}$ By 1953, as prescription of penicillin became more widespread, more than $60 \%$ of $S$. aureus isolates were resistant to penicillin, with development of resistance to other classes of antibiotics such as tetracyclines and macrolides beginning to emerge. $^{6}$ By 1960, despite taking careful surveillance, antibioticresistant $S$. aureus had become the most common cause of hospitalacquired infection. ${ }^{6,7}$ Penicillin-resistant $S$. aureus has been mainly a nosocomial problem until now.

By 1952, introduction of $\beta$-lactam antibiotics for the outgrowth of S. aureus strains, possessing or having acquired $\beta$-lactamases expression ability, led to a resistance rate of $75 \% .^{8}$ The catalytic function of $\beta$-lactamases is the primary cause of resistance to $\beta$-lactam antibiotics. These enzymes hydrolyze the $\beta$-lactam ring in this series of antibiotics, a process that deactivates the drugs. The outgrowth of $\beta$-lactamase producing $S$. aureus led to the commercial development of $\beta$-lactamase-resistant derivatives of penicillin, such as methicillin, possessing a dimethoxybenzoyl side chain that prevents hydrolysis of the $\beta$-lactam ring. But methicillin-resistant $S$. aureus was detected shortly after the introduction of these derivatives.

Over $470 \beta$-lactamases have been recognized to date and are categorized into four distinct functional classes (classes A, B, C and D). ${ }^{9-11}$ Whereas classes A, C and D have evolved dependence on an active-site serine as their key mechanistic feature, class B enzymes are zinc dependent and hence different. The catalytic process resulting in turnover of the former group members involves acylation at the active-site serine by the $\beta$-lactam antibiotic, followed by deacylation of the acyl-enzyme species. It is noteworthy that these enzymes do not share any sequence homologies, structural similarities or mechanistic features with serine or zinc-dependent proteases.

Class A $\beta$-lactamases generally prefer penicillins as substrates, whereas class $\mathrm{C}$ enzymes prefer cephalosporins as the substrate. Class B enzymes can hydrolyze a broad range of substrates including carbapenems, which are resistant to hydrolysis by most of the other classes of enzymes. On the other hand, class D $\beta$-lactamases efficiently hydrolyze oxacillin-type $\beta$-lactams. Classes $A$ and $C \beta$-lactamases are the most common enzymes, among them class A is mostly expressed in S. aureus. ${ }^{12-14}$

Another approach to overcoming resistance mediated by $\beta$-lactamase production has been to combine penicillin with a $\beta$-lactamase inhibitor. Clavulanic acid is one of the favorite inhibitors

\footnotetext{
${ }^{1}$ Department of Laboratory Sciences, School of Paramedical Sciences, Mashhad University of Medical Sciences, Mashhad, Iran; ${ }^{2}$ Student Research Committee, Department of Laboratory Sciences, School of Paramedical Sciences, Mashhad University of Medical Sciences, Mashhad, Iran; ${ }^{3}$ Antimicrobial Resistance Research Center, Mashhad University of Medical Sciences, Mashhad, Iran and ${ }^{4}$ Biotechnology Research Center, Department of Pharmacognosy, School of Pharmacy, Mashhad University of Medical Sciences, Mashhad, Iran

Correspondence: Dr H Sadeghian, Antimicrobial Resistance Research Center, Department of Laboratory Sciences, Mashhad University of Medical Sciences, Mashhad 91967-73117, Iran.

E-mail: sadeghianh@mums.ac.ir

Received 21 August 2013; revised 11 January 2014; accepted 22 January 2014; published online 12 February 2014
} 
used for therapeutic purposes in combination with amoxicillin. Unfortunately, the emergence of clavulanic acid-resistant variants of class A $\beta$-lactamase significantly compromise the efficacy of this combination. A single amino-acid mutation at catalytic position $\operatorname{Ser}^{130}$ (Ser $\rightarrow$ Gly) results in resistance to clavulanate in class A $\beta$-lactamase.

Research for discovery of new $\beta$-lactamase inhibitors to produce the combined antibiotic is now under investigation. In this work, we discovered a new series of natural prenylated coumarins, isolated from Ferula szowitsiana, as class A $\beta$-lactamase inhibitors and showed their potentiating activity on penicillin against some clinical isolates of penicillin-resistant $S$. aureus.

\section{MATERIALS AND METHODS}

\section{Molecular modeling, docking and SAR STUDY}

Structure optimization. Three dimensional structures of the natural coumarins were simulated in Hyper Chem7.5 using the MM+ method (RMS gradient $=0.1 \mathrm{kcal} \mathrm{mol}^{-1}$ ) (HyperChem Release 7, Hypercube Inc., http:// www.hyper.com/). In the second optimization, output files were minimized under Semiempirical AM1 methods (Convergence limit $=0.01$; Iteration limit $=50 ; \quad$ RMS $\quad$ gradient $=0.1 \mathrm{kcal} \mathrm{mol}^{-1} ; \quad$ Polak-Ribiere optimizer algorithm). ${ }^{15}$

Crystal structures of class A and D $\beta$-lactamases (EC 3.5.2.6) were retrieved from RCSB Protein Data Bank (PDB entry: 1BLC and 1K6S, respectively).

Molecular docking. The geometry-minimized structures were docked into the active site of 1BLC and 1K6S with AutoDock Tools version 4.2 (revision 30) $)^{16}$ using the Lamarckian genetic algorithm. ${ }^{17}$ The efficiency of this method to produce bonding models similar to the experimentally results has been reported. ${ }^{18,19}$

The docking regions of $1 \mathrm{BLC}$ and $1 \mathrm{~K} 6 \mathrm{~S}$ were defined with the considering Cartesian chart $4.08,-8.312,-12.31$ and $5.53,13.81,64.95$ as the center of a grid with 52, 44, 63 and 50,50, 50 points in $\mathrm{X}, \mathrm{Y}$ and $\mathrm{Z}$ axes, respectively. The docking parameter files were generated using Genetic Algorithm and Local Search Parameters while the number of generations (Genetic Algorithm and Local Search Parameters runs) and the maximum number of energy evaluations was set to 200 and 2,500,000, respectively (number of individuals in the population $=300$ ). The 200 docked complexes were clustered with a rootmean-square deviation tolerance of $2.0 \AA$. Docking results were submitted to Accelrys DS Visualizer 2.0.1 (http://www.accelrys.com/products/discovery-studio) for further evaluations.

\section{Preparation of $\boldsymbol{\beta}$-lactamase}

Desired bacteria from the exponential growth phase were harvested by centrifugation for $10 \mathrm{~min}$ at $5000 \mathrm{~g}$, washed once and treated with lysozyme in $0.1 \mathrm{M}$ phosphate buffer ( $\mathrm{pH}$ 7.0) to destroy the cell wall. The lysed cells were sonicated at $20 \mathrm{kHz}$ for $10 \mathrm{~min}$. After the cellular debris was removed by centrifugation, the supernatant was used to test for $\beta$-lactamase activity. ${ }^{20}$ One unit of $\beta$-lactamase activity was defined as $1 \mu \mathrm{mol}$ of nitrocefin hydrolyzed.

\section{$\beta$-lactamase inhibitory assessment}

$\beta$-lactamase inhibitory activity was determined according to a previously published study. ${ }^{21,22}$ A 1-mM nitrocefin solution is prepared by dissolving $5.16 \mathrm{mg}$ powder in $0.5 \mathrm{ml}$ dimethyl sulfoxide and then diluting with $9.5 \mathrm{ml}$ of $0.1 \mathrm{~m}$ phosphate buffer ( $\mathrm{pH}$ 7.0). $\beta$-lactamase activity is indicated by measuring the absorbance at $486 \mathrm{~nm}$ for $15 \mathrm{~min}$ after addition of $50 \mu \mathrm{l}$ prepared $\beta$-lactamase $\left(\beta\right.$-lactamase activity $\left.=0.035 \mathrm{U} \mathrm{ml}^{-1}\right)$ to each tube containing inhibitor and nitrocefin $(50 \mu \mathrm{M})$ in $1 \mathrm{ml} 0.1 \mathrm{~m}$ phosphate buffer (incubated at $37^{\circ} \mathrm{C}$ ). The $\mathrm{IC}_{50}$ values were determined from the $\%$ activity of the enzymes at $0.4,2,10,50$ and $250 \mu \mathrm{m}$ concentrations (three replicates for each concentration) of synthetic inhibitors using a sigmoidal dose-response curve in Graphpad Prism 3.0.

Table $1 \boldsymbol{\beta}$-lactamase inhibitory assessment data of the $\boldsymbol{O}$-prenylated coumarins and docking analysis data of the consensus conformers for both class $A(1 B L C)$ and $D(1 K 6 S) \beta$-lactamase

\begin{tabular}{|c|c|c|c|c|c|}
\hline \multirow[b]{2}{*}{ Compound } & \multirow[b]{2}{*}{$1 C_{50}(\mu \mathrm{M})$} & \multicolumn{2}{|c|}{$1 B L C$} & \multicolumn{2}{|c|}{$1 \mathrm{~K} 6 \mathrm{~S}$} \\
\hline & & $K i(\mu M)$ & $\begin{array}{c}\text { Number of } \\
\text { conformers } \\
\text { in cluster }\end{array}$ & $K i(\mu M)$ & $\begin{array}{c}\text { Number of } \\
\text { conformers } \\
\text { in cluster }\end{array}$ \\
\hline 7IPC & $119 \pm 6.1$ & 0.689 & 27 & 147.3 & 33 \\
\hline Umbelliprenin & $54 \pm 2.9$ & 0.173 & 37 & 91.7 & 37 \\
\hline Auraptene & $21 \pm 1.5$ & 0.042 & 34 & 14.1 & 32 \\
\hline Galbanic acid & $47 \pm 3.1$ & 0.087 & 39 & 10.7 & 41 \\
\hline $\begin{array}{l}\text { Clavulanic } \\
\text { acid }\end{array}$ & $24.1 \pm 2.1$ & - & - & - & - \\
\hline
\end{tabular}

The $\mathrm{IC}_{50}$ values are given as \pm s.e. (three replicates for each concentration). The $P$-values of the $\mathrm{IC}_{50}$ data, computed by one-way ANOVA Tukey multiple comparison test are $P<0.001$, except umbelliprenin vs galbanic acid and auraptene vs clavulanic acid $(P>0.05)$.<smiles>CC(C)=C1CCC(C)[C@H](COc2ccc3ccc(=O)oc3c2)[C@@H]1CCC(=O)O</smiles>

Galbanic Acid<smiles>CC(C)=CCC/C(C)=C/CC/C(C)=C/COc1ccc2ccc(=O)oc2c1</smiles>

Umbelliprenin<smiles>CC(C)=CCC/C(C)=C/COc1ccc2ccc(=O)oc2c1</smiles>

Aurapten<smiles>CC(C)=CCOc1ccc2ccc(=O)oc2c1</smiles>

7-Isopentenyloxycoumarin (7IPC)

Figure 1 Chemical structure of the main natural coumarins isolated from F. szowitsiana root. 


\section{Determination of MICs}

The MICs were determined by the tube dilution method, introduced by the National Committee for Clinical Laboratory Standards (NCCLS). ${ }^{23}$ A serial dilution of tested compounds (final concentrations of 400 to $4 \mu \mathrm{M}$ ), were added to the test bacteria in Mueller-Hinton broth and were incubated at $37^{\circ} \mathrm{C}$ for $24 \mathrm{~h}\left(5 \times 10^{5} \mathrm{CFU} \mathrm{ml}^{-1}\right)$. After sufficient incubation $(24 \mathrm{~h})$, the tubes are examined for turbidity indicating growth of the microorganism. For further confidence, the samples were cultured onto Petri dishes containing Muller-Hinton agar $\left(24 \mathrm{~h}\right.$ at $\left.37^{\circ} \mathrm{C}\right)$. The lowest drug concentration of the agent that prevented growth of the test organism was designated the MIC.

\section{及-lactamase inhibitors}

Auraptene, Umbelliprenin and 7IPC were synthesized by reaction of 7-hydroxycoumarin with the appropriate prenyl bromide according to the procedure reported in the previous work. ${ }^{24}$ Galbanic acid was isolated from the air-dried roots of F. szowitsiana according to the previous work. ${ }^{25}$

\section{RESULTS AND DISCUSSION}

In this work we found the ethanolic extract of F. szowitsiana root to have potentiating activity on penicillin bactericidal potency. This was observed using the disc diffusion assay and was confined to $\beta$ lactamase producers, mainly Gram positives. Then the $\beta$-lactamase inhibitory activity of the concentrated extract (final concentrations $=5.0,2.0$ and $1.0 \mathrm{mg} \mathrm{ml}^{-1}$ ) was evaluated against 30 lysed clinical isolates of penicillin-resistant $S$. aureus using penicillin-iodinestarch and nitrocefin tests. ${ }^{26}$ During treating time, 26 samples were not able to hydrolyze penicillin $\mathrm{G}$ in comparison to the control group. Lack of penicillinase activity in these samples indicated the $\beta$-lactamase inhibitory activity of the extract components. The major natural compounds of the extract were three prenylated coumarins: auraptene, umbelliprenin and galbanic $\operatorname{acid}^{25}$ (Figure 1). Afterward, the penicillinase inhibitory activity of the isolated
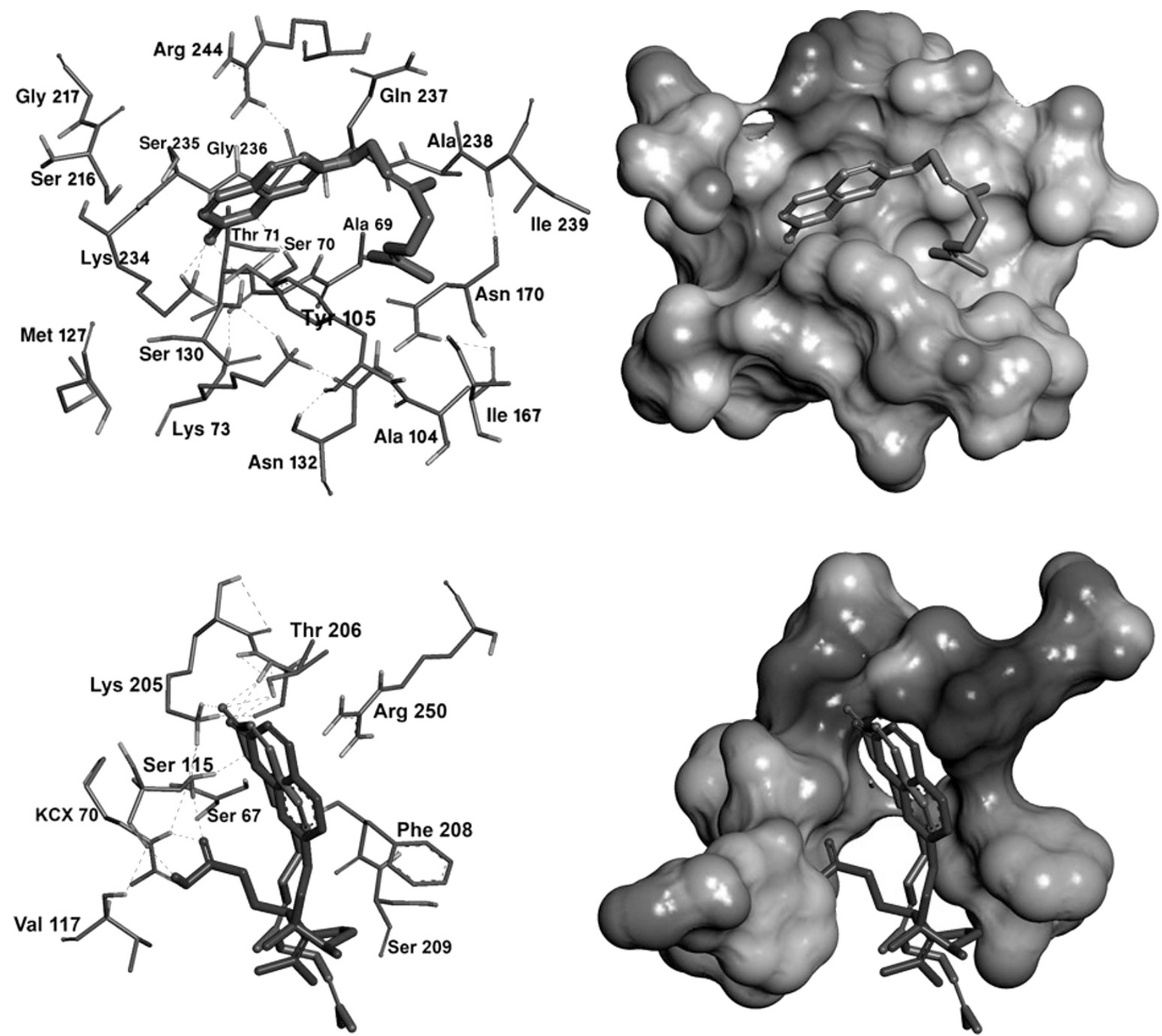

Figure 2 (above) Stick (left) and solvent surface (right) view of the active site pocket residues of 1BLC interacting with auraptene. (below) Stick (left) and solvent surface (right) view of the active site pocket residues of $1 \mathrm{~K} 6 \mathrm{~S}$ interacting with auraptene (green stick) and galbanic acid (blue stick). In this figure hydrogen bonds are shown as dashed line. A full color version of this figure is available at The Journal of Antibiotics journal online. 
coumarins $(100 \mu \mathrm{M})$ was evaluated on the mentioned S. aureus samples by using penicillin-iodine-starch and nitrocefin tests. The compounds inhibited the penicillinase activity in each of these 26 samples.

The $\beta$-lactamase type of the sample bacteria was determined by using the method that had been introduced by Ambler. ${ }^{27}$ Amongst the 30 samples, 26 cases which had shown penicillinase activity belonged to class $\mathrm{A}$ (meticillin resistant) and the remaining belonged to class D. These results imply that the natural coumarin analogs are the class A $\beta$-lactamase inhibitors.

Amongst the 26 samples, one with highest nitrocefin hydrolysis rate was selected for enzyme inhibitory study. $\beta$-lactamase inhibitory potency of the three isolated coumarins was measured in comparison with 7-isopentenyloxycoumarin (7IPC) by using the nitrocefin method (Table 1). ${ }^{21,22}$

Amongst the compounds, auraptene showed the best inhibitory activity $\left(\mathrm{IC}_{50}=21 \pm 1.5 \mu \mathrm{M}\right)$ and the lowest inhibition was observed for 7IPC $\left(\mathrm{IC}_{50}=119 \pm 6.1 \mu \mathrm{M}\right)$. The $\mathrm{IC}_{50}$ of clavulanic acid, a control inhibitor run under the same assay conditions, was $24.1 \pm 2.1 \mu \mathrm{m}$. The $\mathrm{IC}_{50}$ values showed that the coumarin moiety of the test compounds is the pharmacophore for enzyme inhibition and that the prenyl length adversely affects the potency. The results also showed that the

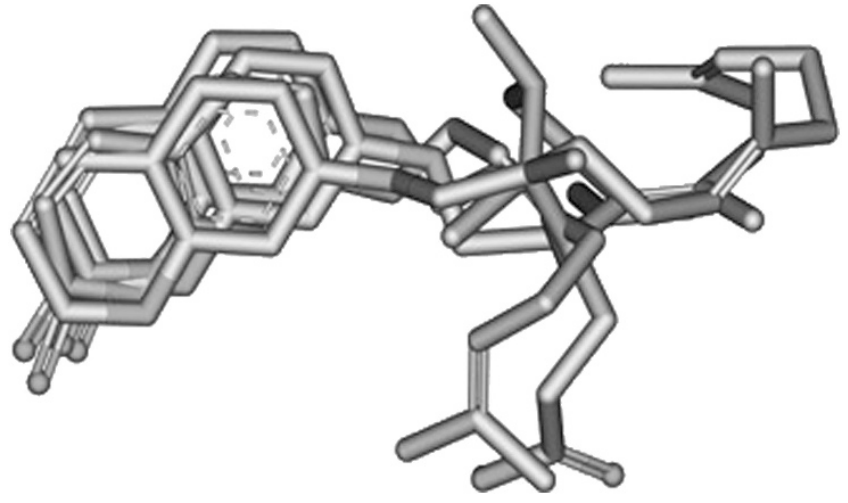

Figure 3 Stick view of the consensus structure of auraptene, umelliprenin galbanic and 7IPC in 1BLC active site pocket. A full color version of this figure is available at The Journal of Antibiotics journal online. structural changes on the farnesyl moiety such as cyclization and carboxylation seen in galbanic acid did not have any effect on its $\beta$-lactamase inhibitory activity (umbelliprenin vs galbanic acid: $P>0.05$; Table 1). For further study of the structure activity relationships, all of the test compounds were docked into the class A $\beta$-lactamase (pdb entry: 1BLC). For each compound, docking analyses showed that most of the docked models (number of conformers in cluster) with the lowest estimated inhibitory constant (Ki) (Table 1) belonged to a cluster in which the coumarin ring was covered by the conserved residues Ser70, Ser130, Lys234, Ser235 and Gly236 (Figure 2). In the desired cluster the residues Ser70, Ser130 and Lys 234 have hydrogen bonds with the lacton portion of the coumarin ring and the prenyl moieties form a hydrophobic interaction with Tyr105, Ile167 and Ile239 (Figure 2). Amongst all of the conformers in the mentioned cluster, the ones with the least $\mathrm{Ki}$ was named as a 'consensus structure' and used for further analyses (Figure 3).

In the mentioned cluster, the side chain of Tyr 105 rotated to make a hydrogen bond with the Tyr129-Ser130 amide bond (Figure 4). Hydrophobic nature of the prenyl moieties of the docked molecules led to formation of this rotation. The new orientation stabilizes the cavity of the bonding pocket (Figure 4 ).

The bonding affinity of the coumarins toward class D $\beta$-lactamase (pdb entry: $1 \mathrm{~K} 6 \mathrm{~S}$ ) was also studied. The docking results showed higher Ki (100-500 folds) for the similar orientations, which had been seen in the selected cluster of 1BLC docking outputs (Table 1).

In this orientation the lacton portion of the coumarin ring had hydrogen bonds with conserved residues Ser115, Lys205 and Thr206 (Figure 2). The steric hindrance prevents hydrogen bond formation with Ser67 unlike that seen for Ser70 in 1BLC. This finding can explain the inactivity of the coumarins against class $\mathrm{D} \beta$-lactamases in S. aureus.

It was interesting that, unlike that seen in prenyl length, the carboxylic moiety had no effect on bonding affinity of galbanic acid toward both enzymes in the desired clusters.

The penicillin potentiating activity of the four compounds $(50 \mu \mathrm{M})$ was tested against all of the penicillinase-positive $S$. aureus (26 cases) by using the serial dilution method according to the NCCLS. The MIC of penicillin G alone averaged $244.2 \pm 12.3$, whereas in individual combination with 7IPC, umbelliprenin, auraptene and galbanic acid the average MICs were significantly different $(P<0.001)$ (Table 2$)$.
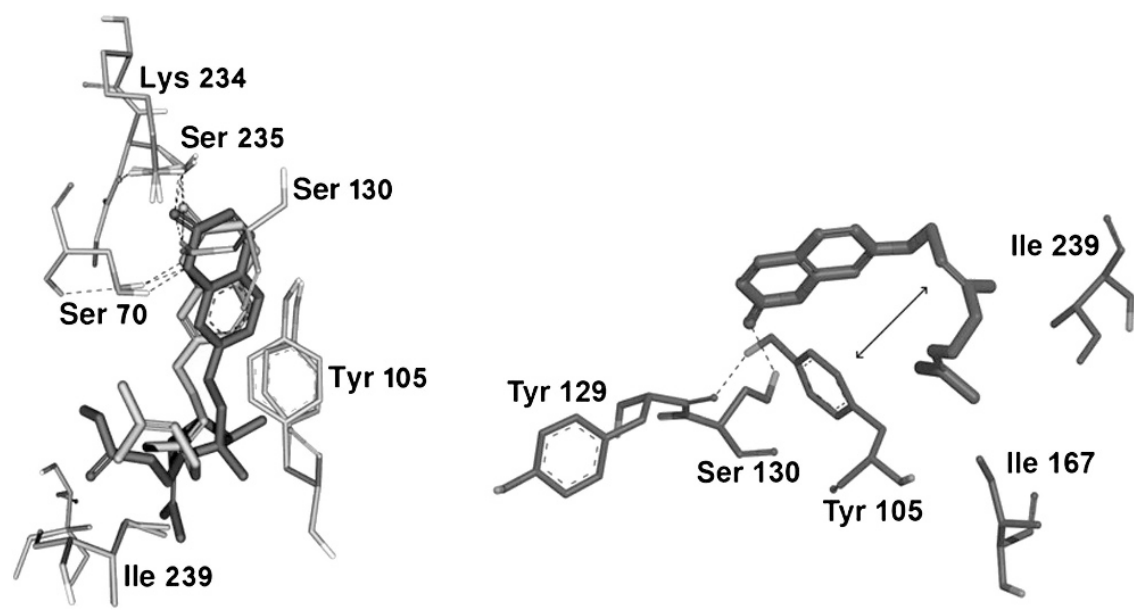

Figure 4 (left) Stick view of consensus structure of auraptene (green stick) and galbanic acid (blue stick) interacting with flexible residue in the active site pocket of 1BLC. (right) hydrophobic interaction of auraptene prenyl moiety with side chain of Ile 167 , Ile 239 and phenyl moiety of Tyr 105 . In this figure hydrogen bonds are shown as dashed line. A full color version of this figure is available at The Journal of Antibiotics journal online. 
Table 2 The average MICs of penicillin G plus 0 -prenylated coumarins $(50 \mu \mathrm{M})$ or clavulanic acid $(50 \mu \mathrm{m})(n=3$ for each bacteria) are given as mean \pm s.e. of 26 individual samples

\begin{tabular}{lcc}
\hline Compound & Average MIC $(\mu \mathrm{m})$ & P-value \\
\hline Penicillin-7IPC & $78.2 \pm 7.3$ & $P<0.001$ \\
Penicillin-umbelliprenin & $21.3 \pm 4.3$ & $P>0.05$ \\
Penicillin-auraptene & $14.2 \pm 4.5$ & $P>0.05$ \\
Penicillin-galbanate & $18.2 \pm 5.6$ & $P>0.05$ \\
Penicillin-clavulate & $10.2 \pm 4.6$ & - \\
Penicillin & $244.2 \pm 12.3$ & $P<0.001$ \\
\hline
\end{tabular}

The MICs of all O-prenylated coumarins alone are $>300 \mu \mathrm{m}$. The P-values were calculated by one-way ANOVA Dunnett's multiple comparison test (comparison between Penicillin-clavulate and other samples).

The MIC of penicillin-clavulate was significantly close to penicillinauraptene, penicillin-galbanate and penicillin-umbelliprenin results $(P>0.05)$ (Table 2). It was interesting that the $O$-prenylated coumarins, themselves, had no antibacterial activity even at high concentrations $(>300 \mu \mathrm{M})$.

The MIC results make the last three natural coumarins candidates for in vivo evaluation against infections caused by resistant $S$. aureus. These compounds could decrease the MIC of penicillin G by 11-16fold. Among the $\beta$-lactam antibiotics, penicillin has been a safe and cheap agent for the treatment of $S$. aureus infections; hence, applying this antibiotic with a suitable $\beta$-lactamase inhibitor could still make it an effective therapeutic agent for curing these infections.

\section{ACKNOWLEDGEMENTS}

We are grateful to the Mashhad University of Medical Sciences for financial support of this work (project 89302).

1 Rubin, R. J. et al. The economic impact of Staphylococcus aureus infection in New York City Hospitals. Emerg. Infect. Dis. 5, 9-17 (1999).

2 Kauffman, C. A., Bradley, S. F. \& Terpenning, M. S. Methicillin-resistant Staphylococcus aureus in long-term care facilities. Infect. Control. Hosp. Epidemiol. 11, 600-603 (1990).

3 Storch, G. A., Radcliff, J. L., Meyer, P. L. \& Hinrichs, J. H. Methicillin-resistant Staphylococcus aureus in a nursing home. Infect. Control. 8, 24-29 (1987).

4 Levine, D. P., Cushing, R. D., Jui, J. \& Brown, W. J. Community-acquired methicillinresistant Staphylococcus aureus endocarditis in the Detroit Medical Center. Ann. Intern. Med. 97, 330-338 (1982).

5 Mulligan, M. E. et al. Methicillin-resistant Staphylococcus aureus: A consensus review of the microbiology, pathogenesis, and epidemiology with implications for prevention and management. Am. J. Med. 94, 313-328 (1993).
6 Bradley, S. F. Methicillin-resistant Staphylococcus aureus infection. Clin. Geriatr. Med. 8, 853-868 (1992).

7 Wise, R. I., Ossman, E. A. \& Littlefield, D. R. Personal reflections on nosocomial staphylococcal infections and the development of hospital surveillance. Rev. Infect. Dis. 11, 1005-1019 (1989).

8 Finland, M. Changing patterns of resistance of certain pathogenic bacteria to antimicrobial agents. New Engl. J. Med. 252, 570-580 (1955).

9 Ambler, R. P. The structure of beta-lactamases. Philos. Trans. R Soc. Lond. B Biol. Sci. 289, 321-331 (1980)

10 Jaurin, B. \& Grundstrom, T. ampC cephalosporinase of Escherichia coli K-12 has a different evolutionary origin from that of $\beta$-lactamases of the penicillinase type. Proc. Natl Acad. Sci. USA 78 (8 I), 4897-4901 (1981).

11 Ouellette, M., Bissonnette, L. \& Roy, P. H. Precise insertion of antibiotic resistance determinants into Tn21-like transposons: nucleotide sequence of the OXA-1 betalactamase gene. Proc. Natl Acad. Sci. USA 84, 7378-7382 (1987).

12 Beceiro, A., Bou, G. \& Class, C. $\beta$-Lactamases: an increasing problem worldwide. Rev. Med. Microbiol. 15, 141-152 (2004).

13 Bush, K. \& Mobashery, S. How $\beta$-lactamases have Driven Pharmaceutical Drug Discovery. Resolving the Antibiotic Paradox (Springer-Verlag, New York, 1998).

14 Yang, Y., Rasmussen, B. A., Shlaes, D. M. \& Class, A. $\beta$-lactamases-enzyme-inhibitor interactions and resistance. Pharmacol Ther. 83, 141-151 (1999).

15 Seyedi, S. M. et al. Design, synthesis and SAR studies of 4-allyoxyaniline amides as potent 15-lipoxygensae inhibitors. Bioorg. Med. Chem. 17, 1614-1622 (2009).

16 Morris, G. M. et al. Software news and updates AutoDock4 and AutoDockTools4: automated docking with selective receptor flexibility. J. Comput. Chem. 30, 2785-2791 (2009)

17 Morris, G. M. et al. Automated docking using a Lamarckian genetic algorithm and an empirical binding free energy function. J. Comput. Chem. 19, 1639-1662 (1998).

18 Jabbari, A. et al. Synthesis and SAR studies of 3-allyl-4-prenyloxyaniline amides as potent 15-lipoxygenase inhibitors. Bioorg. Med. Chem. 20, 5518-5526 (2012).

19 Nikpour, M. et al. Design, synthesis and biological evaluation of 6-(benzyloxy)-4methylquinolin-2 $(1 \mathrm{H})$-one derivatives as PDE3 inhibitors. Bioorg. Med. Chem. 18, 855-862 (2010)

20 Sadeghian, H. et al. Design, synthesis, and structure-activity relationship study of 5 amido-1-(2,4-dinitrophenyl)-1H-4-pyrazolecarbonitrils as DD-carboxypeptidase/penicillin-binding protein inhibitors with Gram-positive antibacterial activity. Med. Chem. Res. 19, 103-119 (2010).

21 Bethel, C. R., Hujer, A. M., Helfand, M. S. \& Bonomo, R. A. Exploring the effectiveness of tazobactam against ceftazidime resistant Escherichia coli: insights from the comparison between susceptibility testing and $\beta$-lactamase inhibition. FEMS Microbiol. Lett. 234, 99-103 (2004).

22 Livermore, D. M. \& Brown, D. F. J. Detection of $\beta$-lactamase-mediated resistance. J. Antimicrob. Chemother. 48(Suppl 1), 59-64 (2001).

23 Sadeghian, A., Pordel, M., Safdari, H., Fahmidekar, M. A. \& Sadeghian, H. 11-Chloro3-methyl-3H-imidazo[4,5-A]acridine (CMIA) as a potent and selective antimicrobial agent against clinical isolates of highly antibiotic-resistant Acinetobacter baumannii. Med. Chem. Res. 21, 3897-3901 (2012).

24 Iranshahi, M. et al. Synthesis and SAR studies of mono O-prenylated coumarins as potent 15-lipoxygenase inhibitors. Eur. J. Med. Chem. 57, 134-142 (2012).

25 Iranshahi, M. et al. Sesquiterpene coumarins from Ferula szowitsiana and in vitro antileishmanial activity of 7-prenyloxycoumarins against promastigotes. Phytochemistry 68, 554-561 (2007).

26 Skinner, A. \& Wise, R. A comparison of three rapid methods for the detection of beta-lactamase activity in Haemophilus influenzae. J. Clin. Pathol. 30, 1030-1032 (1977).

27 Ambler, R. P. et al. A standard numbering scheme for the class A $\beta$-lactamases [1]. Biochem. J. 276, 269-270 (1991). 